\section{INTERFACES}

\section{Phuse Energies}

from our Materials Science Correspondent

As men living at the interface of the earth and air, as scientists living at the interface of the prosaic and the mysterious, as travellers scowling at customs men, we cannot escape interfaces. Certainly the detailed description of interfaces has been a sustained concern of scientists of many kinds; materials scientists, for instance, have long studied the morphology, composition and energies of interfaces between crystals or between phases. An exact knowledge of interfacial energies, in particular, is a precondition of understanding the behaviour of interfaces in systems undergoing transitions, and a particularly important category of transition is the homogeneous nucleation and growth of a stable phase at the expense of a metastable one.

According to Volmer, Becker and Döring's classical theory of homogencous nucleation, an embryo of the stable phase becomes viable and grows steadily only when, by a chance fluctuation, it comes to exceed a critical spherical size. This size is such that a further atom, in deserting the metastable for the stable phase, relcases in volume free energy more than the interfacial energy needed for the nucleus to increase in size. The theory has long been accepted as a central tenet of the theory of phase transformation (and of freezing of liquids in particular), but it has never been rigorously confirmed by experiment. The nearest approach, perhaps, was an attempt by Sundquist and Oriani in 1962 to relate critical undercooling in a two-liquid miscibility gap to the known interfacial energy. Subsequent criticism, however, casts doubt on the reliability of their conclusions. A. E. Nielsen and S. Sarig (J. Cryst. Growth, 8, 1; 1971) have now published results, obtained by means of an ingenious technique, which really seem to constitute a quantitative confirmation of the Volmer-Becker-Döring theory. They studied nucleation of droplets of second phase in a ternary miscibility gap in the methanol-watertribromomethane system. Two liquids were mixed rapidly and completely at a rapid flow rate, and the time $\tau$ to onset of turbidity was measured to a factor of a millisecond. This delay time constitutes an induction period for droplet nucleation plus diffusion controlled growth to a size large enough to scatter light to a detectable degree. Because the supersaturation of the mixed liquid is known, and the volume fraction of new phase is small enough to have negligible effect on the composition of the parent liquid, all terms are known in the Volmer equations except the interfacial energy $\sigma$ : thus $\sigma$ can be calculated from the measured $\tau$ values for different compositions and supersaturations of the parent phase. Nielsen and Sarig compared these $\sigma$ values with values measured directly by means of the classical drop weight method and obtained good agreement for the two sets of $\sigma$ values, subject to a correction made for the variation of $\sigma$ with surface curvature for very small droplets. This agreement was obtained for delay times less than $1 \mathrm{~ms}$, corresponding to large supersaturations. Longer times corresponded to anomalous behaviour which Niclsen and Sarig attribute to heterogeneous nucleation.

Interfacial energies in another context have been considered by G. H. Bishop, W. H. Harth and G. A. Bruggeman (Acta Metall., 19, 37; 1971). These

workers have examined the morphology of grain boundaries in zinc bicrystals of controlled orientations, and have found that for certain narrow ranges of misorientation (close to but not identical with twin orientations) the boundary became stably faceted ...-that is it did not strive to achieve a state of minimum total area. The nature of the "energy cusps" in the Wulff plot, which expresses grain boundary energy as a function of grain boundary orientation for a fixed mutual orientation of the contiguous lattices, was analysed in detail from the observed boundary morphologies: the determination of these cusps is an important achievement at a time when interest in grain boundary structure has suddenly revived after a long period of quiescence.

\title{
Radio Prediction of Solar Profon Events
}

A SOLAR flare heralds a violent outburst of energy from the Sun which produces radiation extending from radio wavelengths, through optical, to X-radiation as well as high energy protons and slower moving plasma clouds. The X-rays and microwave bursts reach the Earth almost simultaneously with the light, but the more energetic protons arrive after times ranging from a few tens of minutes to several hours. The low energy protons and the plasma cloud reach the Earth and interact with the upper atmosphere 1 or 2 days later still. D. L. Croom has been investigating methods of predicting these solar proton events and he reports his findings in next Monday's Nature Physical Science.

The study of the proton streams associated with solar flares is important for two principal reasons. First, these relatively rare events are little understood. It is known, for example, that relativistic protons with energies between 1 and 60 $\mathrm{GeV}$ actually reach the surface of the Earth and that lower energy protons of from $30-300 \mathrm{MeV}$ can penetrate into the ionosphere at the poles and produce absorption of radio communications. But much more information is needed about the phenomena which are so effective in accelerating particles to these high energies and the mechanisms which can contain both high and low energy protons in the solar corona and the interplanetary medium for such a wide range of times. Clearly some warning of the onset of such events would be a great advantage in preparing equipment, particularly the launching of balloons and rockets for the detection of the less energetic particles high in the Earth's atmosphere.

The second reason for interest is that these proton events may have an effect on man. The most intense solar proton events reaching the Earth's surface may exceed the constant galactic background cosmic ray level by a factor of up to ten for a few tens of minutes; this will have an integrated effect on the surface of the Earth which adds little to cosmic ray dose received by man. But outside the Earth's protective atmosphere the effects of the flux of lower energy protons which lasts much longer and occurs more frequently cannot be dismissed so readily. Plans for manned space flight, and particularly extravehicular activity on the Moon and planets, must obviously include some precautionary avoiding action; similar steps may be necessary for high level flying in aircraft such as Concorde. Any warning of such a proton event which can be given by optical or radio observations is therefore highly desirable.

For about 10 years it has been known that a particular kind of radio emission associated with solar flares, known as type IV activity, is connected with proton events. This activity is a continuum which can extend right through the radio spectrum from decametre to millimetre wavelengths and is caused by synchrotron emission from electrons emitted at the time of the ejection of the high energy protons. Although all proton events are associated with type IV activity, the reverse is not true--not all type IV events are followed by proton events and they are therefore not a reliable guide. Croom now claims to have found a more reliable method of predicting solar proton events using detailed spectral characteristics of type IV events. Following up ideas put forward by Castelli, Aarons and Michael (J. Geophys. Res.. 72,5491 ; 1967), Croom has found that centimetre and millimetre wave observations are critical in setting up criteria for distinguishing proton and non-proton associated type IV events. 